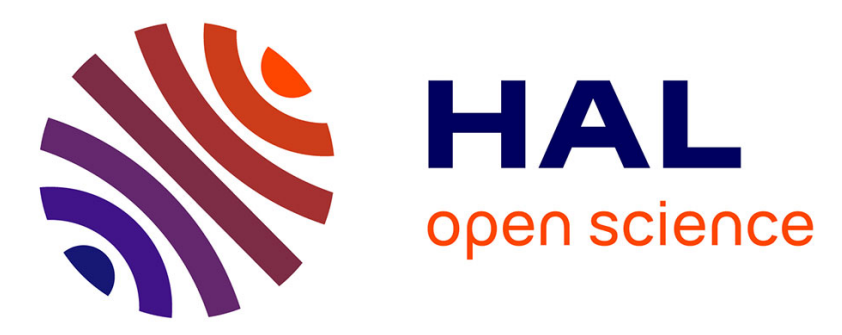

\title{
Information Spatial focusing scheme for UWB Wireless Communications in Smart Environments
}

Theodoros Mavridis, Julien Sarrazin, Luca Petrillo, Philippe de Doncker, Aziz Benlarbi-Delai

\section{- To cite this version:}

Theodoros Mavridis, Julien Sarrazin, Luca Petrillo, Philippe de Doncker, Aziz Benlarbi-Delai. Information Spatial focusing scheme for UWB Wireless Communications in Smart Environments. Antennas and Propagation Society Newsletter, IEEE, 2015, 14, pp.20 - 23. 10.1109/LAWP.2014.2354059 . hal-01069247

\section{HAL Id: hal-01069247 \\ https: / hal.sorbonne-universite.fr/hal-01069247}

Submitted on 29 Sep 2014

HAL is a multi-disciplinary open access archive for the deposit and dissemination of scientific research documents, whether they are published or not. The documents may come from teaching and research institutions in France or abroad, or from public or private research centers.
L'archive ouverte pluridisciplinaire HAL, est destinée au dépôt et à la diffusion de documents scientifiques de niveau recherche, publiés ou non, émanant des établissements d'enseignement et de recherche français ou étrangers, des laboratoires publics ou privés. 


\title{
Information Spatial focusing scheme for UWB Wireless Communications in Smart Environments
}

\author{
Theodoros Mavridis, Student Member, IEEE, Julien Sarrazin, Member, IEEE, Luca Petrillo, Philippe De Doncker, \\ Aziz Benlarbi-Delaï
}

\begin{abstract}
This letter presents an information spatial focusing method for future Smart Environments using UWB Wireless communications. This method allows to send high data rate information at predetermined specific spatial positions without localizing users, thereby protecting their privacy. The proposed approach is a combination of simplified Ultra Wide Band (UWB) Beamforming and signal processing. Compared to classical UWB Beamforming, the proposed method exhibits greater information focusing capabilities by increasing greatly the peak-to-floor ratio between main and side beams.
\end{abstract}

Index Terms-Beamforming, Smart Environments, UWB, spatial, focusing, localization

\section{INTRODUCTION}

The Internet of Things (IoT) [1], [2] and Smart Environments (SE) [3] appear to be the next revolution in wireless communications. The emerging technologies, such as Ultra-Wideband (UWB), will soon allow reliable and high-speed communications to provide new experience to users such as augmented reality, video streaming, Gigabit Ethernet. A key issue of such SEs will be the capability to spatially localize the users, or to spatially confine the informations in indoor environments. Localization methods have been widely studied [4] and are particularly efficient. However, permanent localization of the users lead to privacy issues. Another solution is to confine communication within a spatial area. This non-intrusive geocasting scheme allows to create real SE's interacting with the users. To achieve this, a first approach is to use Beamforming (BF) or Time Reversal (TR) in order to spatially confine the transmitted electromagnetic wave in a given area.

Beamforming has been widely studied and implemented in different scenarios [5], [6]. The main advantages of BF is the simple physical understanding of the method and the great performances. However, these focusing performances (beamwidth) require large arrays of antennas and large number of antennas. Since the inter-antenna distance is fixed by the carrier wavelength, the beamwidth highly depends on the array size and then on the number of antennas. Since in UWB, the wavelength is about $4.5 \mathrm{~cm}$ at center frequency, this involves large arrays complicated to implement in indoor

T. Mavridis, L. Petrillo and P. De Doncker are with OPERA Dpt. Wireless Communications Group, Université Libre de Bruxelles (ULB), B1050 Brussels, Belgium e-mail: (tmavridi@ulb.ac.be ; lpetrillo@ulb.ac.be ; pdedonck@ulb.ac.be)

T. Mavridis, J. Sarrazin and A. Benlarbi-Delaï are with L2E Dept., Université Sorbonne Universits, UPMC Univ Paris 06, UR2, L2E, F-75005, Paris, France e-mail: (julien.sarrazin@upmc.fr; aziz.benlarbi_delai@umpc.fr) environment. Furthermore, to allow beam steering, it is necessary to implement variable phase shifters and amplifiers at each antenna. This creates costly systems.

Time Reversal [7] is the second solution used for spatial focusing and it is particularly efficient with large bandwidths. The method used the multipath components (MPCs) created by the environment to achieve spatial and temporal focusing. It has been shown that the method works better in non line-of-sight (NLOS) configurations. Also, it is a very expensive solution since it requires to be able to sample the impulse response and to reverse it over time [7].

To be effectively implemented, only low-cost solutions have to be chosen. Consequently, there is an interest to develop new methods using simple and cheap systems to spatially confine communication.

To reduce the required number of antennas, a solution is to use Wideband Beamforming (WBF) which allows to have sparse arrays. Indeed, the inter-antenna distance is no longer reduced to the half of the wavelength [8]. However, the beamwidth still highly depends on the number of antennas.

The idea we propose to overcome this issue in BF is to consider a cooperative receiver. Instead of trying to spatially focus the energy as it is done classicaly in BF, it is proposed to spatially confine communication at the information level.

Section II presents the considered scenario of Smart Environments. Then, section III describes the new two-antenna scheme to focus information. An experimental validation of the method is presented in section IV and a comparison with narrowband beamforming is conducted in section V. Finally, section VI concludes this communication.

\section{SCENARIO}

In Smart Environments, several objects in an environment are connected with relevant data. Classically, beamforming is proposed to achieve spatial focusing. In Fig. 1, a schematic beamformer is presented to focus the energy in two directions. The discrimination of the two objects is conditioned on the width of the beam described by the half-power beamwidth $\Delta \theta_{-3 \mathrm{~dB}}$. But this value is determined by the array size and so by the number of antennas.

To overcome this issue, this letter imposes a two-antenna array. The scheme presented in Fig. 2 shows a two-antenna transmitting base station having hence a large radiation pattern. 


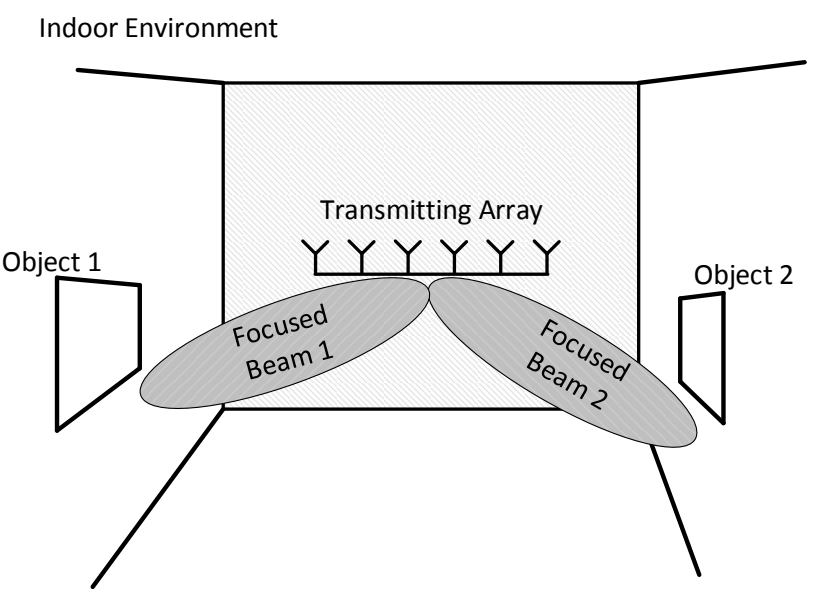

Fig. 1. The Beamformer array is used to focuse the energy in a beams 1 and 2 related to the objects 1 and 2 .

Discrimination between objects 1 and 2 is not achieved by focusing energy but rather by making information 1 decodable only at position 1 , and information 2 only at position 2 .

\section{Novel Two-Antenna Scheme}

\section{A. Two-antenna UWB Beamforming system}

The proposed system uses an UWB transmitter equipped with two antennas spaced by a distance $D$. One antenna has a variable delay line $\tau_{p}$. The baseband transmitted signal is $s(t)$ at the first antenna, and $e^{j \omega_{c} \tau_{p}} s\left(t-\tau_{p}\right)$ at the second one. $s(t)$ is assumed to be an UWB baseband pulse and $\omega_{c}$ is the carrier angular frequency. The baseband received signal

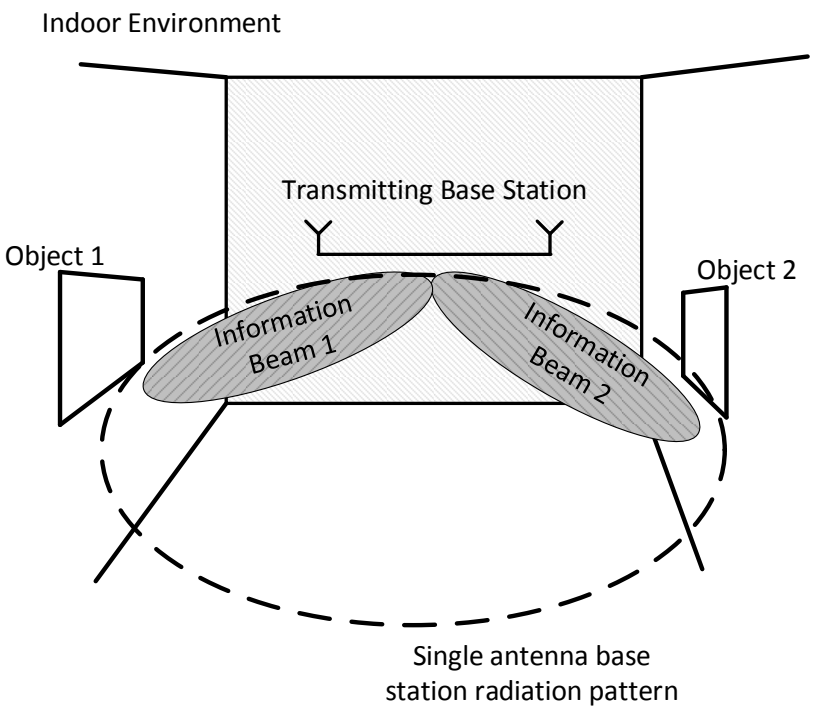

Fig. 2. The base station radiation pattern shows the energy spread while the informations are only decodable in the Information Beams 1 and 2 related to the objects 1 and 2 . depending on time $t$ and angular position $\theta$ is noted

$$
y(t, \theta)=h_{1} \otimes s(t)+h_{2} \otimes e^{j \omega_{c} \tau_{p}} s\left(t-\tau_{p}\right)
$$

where $\otimes$ denotes the convolution product, $h_{1}$ and $h_{2}$ are the channel impulse responses between receiving $\mathrm{Rx}$ and, respectively, transmitting antennas $\mathrm{Tx}_{1}$ and $\mathrm{Tx}_{2}$. Kaiser [8] has introduced the beampattern or the total energy over the bandwidth $E(\theta)$ by the following definition:

$$
E(\theta)=\int_{-\infty}^{\infty}|y(t, \theta)|^{2} d t
$$

where $\theta$ is the angular position of the receiver with respect to the center of the transmitting array as presented in Fig. 3 . To steer $E(\theta)$ in a particular direction $\theta_{p}$, the delay has to be adjusted as:

$$
\tau_{p}=\frac{D}{c} \sin \theta_{p}
$$

where $c$ is the speed of light. By sending each signal with the right delay $\tau_{p}$, it is possible to receive each signal at the right location. However, for sake of simplicity, in the following, it is assumed that only a single signal is transmitted.

It has been shown in [8] that the peak-to-floor ratio of $E(\theta)$ depends on the number of antennas and is limited to $3 \mathrm{~dB}$ for a two-antenna array. To increase focusing capabilities, a larger number of antennas is required.

\section{B. Proposed two-antenna scheme}

To increase the peak-to-floor ratio and the receiver's SNR, a time-sequencing method is used with a classical beamforming system. This method is carried out in two steps. In a first step, the signal $s(t)$ is sent to the two antennas with a delay $\tau_{p}$ applied on antenna 2. The received signal is $y_{0}(t)$. In a second step, the signal on antenna 2 is also phase shifted by $\pi$. The received signal is $y_{\pi}(t)$. The same data is consequently sent twice, thereby dividing the achievable data rate by two. The receiver then recombines the two received signals according to:

$$
\mathrm{BP}_{\text {equ }}(\theta)=\int_{-\infty}^{\infty}\left|y_{0}(t, \theta)\right|^{2}-\left|y_{\pi}(t, \theta)\right|^{2} d t .
$$

$\operatorname{BP}_{\text {equ }}(\theta)$ is maximal in a given direction $\theta_{p}$. A functional scheme of the proposed method is shown in Fig. 3. It is to be noted that the receiver itself remains a simple envelope detector as in the case of pulse-based communication. The added complexity here lies in the clock $\mathrm{T}$ that needs to be synchronized with the transmitter. The clock speed however can be much slower than the bit rate and can be therefore implemented in the preamble of the transmitted data frames. The limitation here comes from the channel. In fact, the clock $\mathrm{T}$ should be fast enough for the receiver to receive twice the same data with an invariant channel (once during the in-phase step and one during the out-of-phase step). Hence, T must be small with respect to the channel's time coherence.

For the sake of simplicity, the channel is supposed to be lineof-sight only. The distance between the Tx array and the Rx is large enough with respect to $D$ to assume the same magnitude 


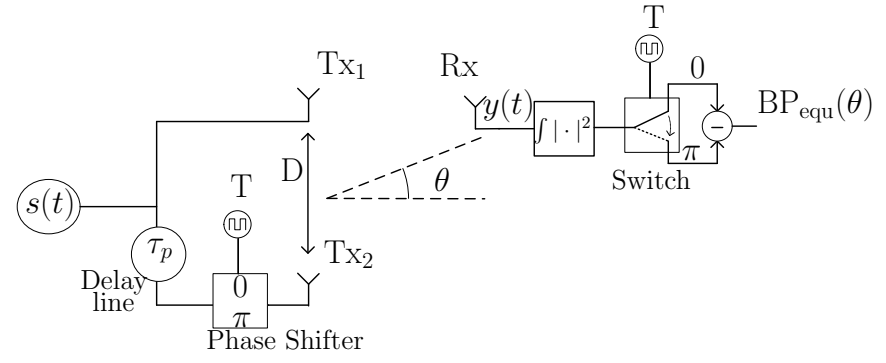

Fig. 3. Proposed spatial focusing system scheme.

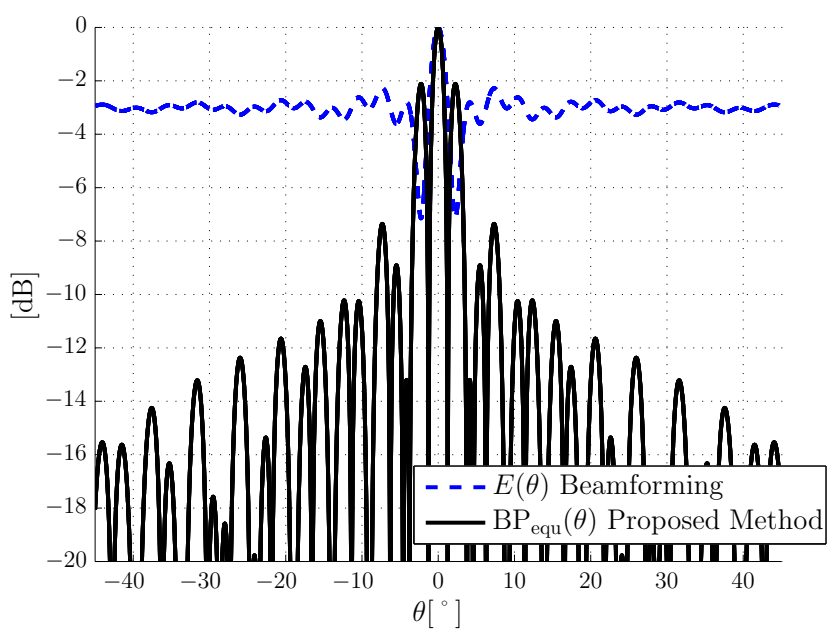

Fig. 4. Comparison between $E(\theta)$ for the two-antenna UWB Beamforming system and $\mathrm{BP}_{\text {equ }}(\theta)$ for the proposed method. The system has a $D=50$ cm, $\theta_{p}=0$, carrier frequency $f_{c}=6.85 \mathrm{GHz}$, bandwidth $\Delta f=7.5 \mathrm{GHz}$ and a Nyquist emitted pulse. The results have been normalized with respect to the maximum value.

for $h_{1}$ and $h_{2}$. This magnitude is normalize to unity, so that [9]:

$$
\begin{aligned}
& h_{1}=e^{j \omega_{c} \tau_{1}} \delta\left(t-\tau_{1}\right) \\
& h_{2}=e^{j \omega_{c} \tau_{2}} \delta\left(t-\tau_{2}\right)
\end{aligned}
$$

where $\tau_{i}$ is the propagation delay between transmitter $\operatorname{Tx}_{i}$ and the receiver Rx with $i=1,2, \omega_{c}=2 \pi f_{c}, f_{c}$ is the carrier frequency and $\delta($.$) is the Dirac delta function.$

$$
\begin{aligned}
y_{0}(t, \theta)= & e^{j \omega_{c} \tau_{1}} \delta\left(t-\tau_{1}\right) \otimes s(t) \\
& +e^{j \omega_{c} \tau_{p}} e^{j \omega_{c} \tau_{2}} \delta\left(t-\tau_{2}\right) \otimes s\left(t-\tau_{p}\right) \\
y_{\pi}(t, \theta)= & e^{j \omega_{c} \tau_{1}} \delta\left(t-\tau_{1}\right) \otimes s(t) \\
& -e^{j \omega_{c} \tau_{p}} e^{j \omega_{c} \tau_{2}} \delta\left(t-\tau_{2}\right) \otimes s\left(t-\tau_{p}\right)
\end{aligned}
$$

It can be easily shown with (6) that:

$$
\mathrm{BP}_{\text {equ }}(\theta)=2 \int_{-\infty}^{\infty} \cos \omega_{c} \Delta \tau s\left(t-\tau_{1}\right) s\left(t-\tau_{p}-\tau_{2}\right) d t
$$

with $\Delta \tau=\tau_{p}+\tau_{2}-\tau_{1}=\frac{D}{c}\left(\sin \theta+\sin \theta_{p}\right)$. The equivalent beampattern BP of the sequenced two-antenna system is ploted in Fig. 4. From Fig. 4, it can be inferred that the peakto-floor ratio has been widely increased for the equivalent beampattern (after signal processing) as compared to the classical beamforming scheme.
The equations have been developed here for line-of-sight channels. However, in real environments, channels are made up of several multipath components (MPCs). By integrating the received signals as in (7), it can be shown that the effect of MPCs is highly reduced due to their high delay.

\section{EXPERIMENTAL Focusing PERFormanCE}

To assess the validity of the proposed method in an indoor environment, an experiment in a $5 \mathrm{~m} \times 4 \mathrm{~m} \times 3 \mathrm{~m}$ room has been performed using a Vector Network Analyser (VNA). The distance $D$ between the transmitting antennas was fixed to $50 \mathrm{~cm}$ and the receiving antenna was placed at $1.5 \mathrm{~m}$ from the transmitting array and was moved with an automatic positioning device. UWB SkyCross SMT-3TO10M-A omnidirectional antenna have been used for both transmitter and receiver. The total energy $E(\theta)$ given by (2), and the equivalent beampattern $\mathrm{BP}_{\text {equ }}$, see eq. (7), are obtained by post-processing and compared by considering that $s(t)$ is a Nyquist pulse with bandwidth $\Delta f$. Measurements have been conducted in the UWB band (3.1-10.6 GHz) and are plotted in Fig. 5.

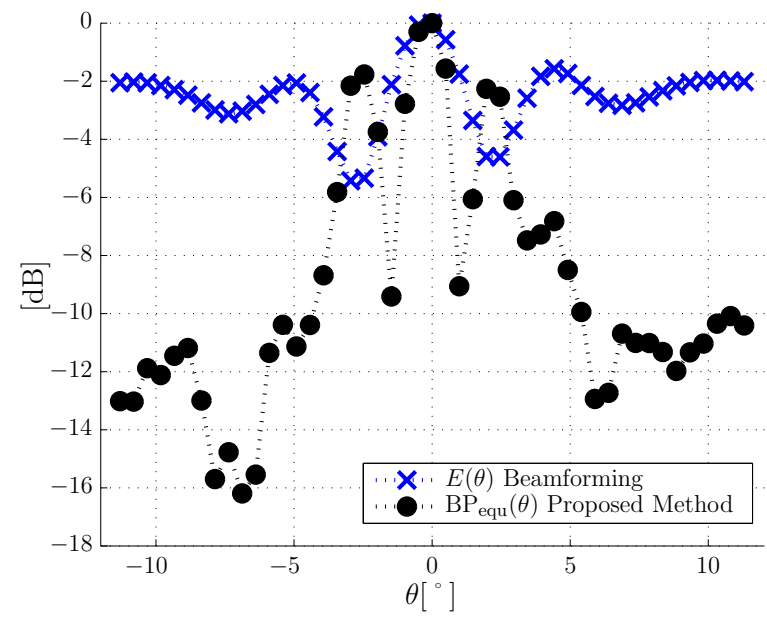

Fig. 5. Comparison between $E(\theta)$ for the experimental results of the beamforming and $\mathrm{BP}_{\text {equ }}(\theta)$ for the proposed method in an indoor environment. The system has a $D=50 \mathrm{~cm}, \theta_{p}=0$, carrier frequency $f_{c}=6.85 \mathrm{GHz}$, bandwidth $\Delta f=7.5 \mathrm{GHz}$ and a Nyquist emitted pulse. The results have been normalized with respect to the maximum value.

Even if the proposed method is developed under free-space assumption (7), it is clear by observing Fig. 5 that it still works in a standard indoor environment. It is important to notice that the $\theta$-axis of this figure goes from $-11^{\circ}$ to $11^{\circ}$. It is also shown that the $\mathrm{BP}_{\text {equ }}$ drops below $-10 \mathrm{~dB}$ after $5^{\circ}$. Since a classical beamformer cannot achieve a beam defined by $-3 \mathrm{~dB}$ criteria with only two antennas, the comparison between the two methods is not straightforward. However, it can be basically concluded that a two-antenna classical beamformer is not able to spatially focus the signal (since there is only $2 \mathrm{~dB}$ of peak-to-floor ratio) while the proposed method can overcome this bottleneck. 
The focusing performance are evaluated by studying the beamwidth at $-3 \mathrm{~dB}: \Delta \theta_{-3 \mathrm{~dB}}$. With the proposed scheme, it can be seen in Fig. 6 that the beamwidth decreases with increasing bandwidth $\Delta f$ and array size $D$. To reach a given performance

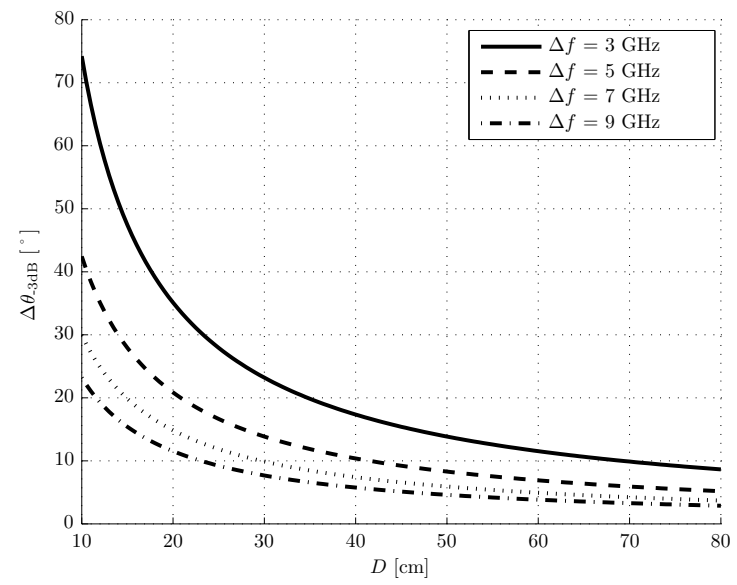

Fig. 6. Beamwidth at $-3 \mathrm{~dB}$ with array size $D$ and a Nyquist pulse on a bandwidth $\Delta f$ at $f_{c}=6.85 \mathrm{GHz}$.

(determined by $\Delta \theta_{-3 \mathrm{~dB}}$ ), a trade-off between the array size and the bandwidth has to be chosen. In practical situations, the array size is physically set. Then, the bandwidth has to be adjusted to reach the required performances.

\section{COMPARISON WITH NARROWBAND BEAMFORMING}

The proposed method can be compared to narrowband linear array beamformers.

Assuming that the beamformer operates at a center frequency $f_{0}$ and that antennas are separated by half a wavelength, the total number of antennas $\mathrm{N}_{\text {Beamformer }}$ is then directly related to the size of the array. In this section, we compare, for a given performance (that is a given $\Delta \theta_{-3 \mathrm{~dB}}$ ), the narrowband beamformer in terms of $\mathrm{N}_{\text {Beamformer }}$ to the proposed two-antenna UWB beamformer scheme in terms of required frequency bandwidth.

TABLE I

COMPARISON BETWEEN A CLASSIC BEAMFORMER AND THE PROPOSED METHOD. PARAMETERS ARE CALCULATED SUCH AS THE TWO SCENARIOS HAVE THE SAME TOTAL SIZE $D$ AND BEAMWIDTH AT -3 DB

\begin{tabular}{ccccc}
\hline \hline$f_{0}[\mathrm{GHz}]$ & $D[\mathrm{~cm}]$ & $\Delta \theta_{-3 \mathrm{~dB}}\left[{ }^{\circ}\right]$ & $N_{\text {Beamformer }}$ & $\Delta f[\mathrm{GHz}]$ \\
\hline \multirow{3}{*}{3} & 20 & 26.33 & 4 & 2.75 \\
& 40 & 12.81 & 8 & 2.73 \\
& 60 & 8.57 & 12 & 2.74 \\
& 80 & 6.39 & 16 & 2.73 \\
\hline \multirow{3}{*}{5} & 20 & 15.45 & 7 & 4.56 \\
& 40 & 7.65 & 13 & 4.54 \\
& 60 & 5.13 & 20 & 4.56 \\
& 80 & 3.87 & 27 & 4.57 \\
\hline \multirow{3}{*}{7} & 20 & 10.98 & 9 & 6.37 \\
& 40 & 5.48 & 19 & 6.36 \\
& 60 & 3.64 & 28 & 6.35 \\
\hline \hline
\end{tabular}

Table I shows the parameters of the two scenarios. For different center frequencies and array sizes, the half-power beamwidth is given, as well as the required number of antennas $\mathrm{N}_{\text {Beamformer }}$ and the required bandwidth, for the narrowband beamformer and the proposed UWB beamformer, respectively. It is well known that for a given center frequency, the array size needs to be increased in order to obtain a narrower beamwidth. In the case of classical narrowband beamformers, this results in a greater number of antennas and phase shifters. Thus, obtaining steerable narrow beams is complicated and involves complex systems. In the case of our proposed method, the number of antennas remains equal to two and the required bandwidth does not change significantly with the array size. Consequently, for a given center frequency, decreasing the beamwidth is only a matter of antenna location.

It can be seen that the method can reach narrow beamwidths with only two antennas. This simplifies a lot the transmitting array of the beamformer since it allows to save phase shifters and complex signal processing.

\section{CONCLUSION}

The system presented in this letter allows to spatially focus information with a high peak-to-floor ratio with only two transmit antennas. The system, based on a two-antenna UWB beamforming architecture, consists in a time sequencing at the transmitting side and a recombination step at the receiving side, which is assumed to be coopering and synchronized. The improved system allows to reach a peak-to-floor of 15 $\mathrm{dB}$ while it was limited to $3 \mathrm{~dB}$ in a typical two-antenna beamforming system. This improvement is done at the expense of the data rate which is divided by 2 due to time-sequencing.

\section{REFERENCES}

[1] J. Gubbi, R. Buyya, S. Marusic, and M. Palaniswami, "Internet of things (iot): A vision, architectural elements, and future directions," Future Generation Computer Systems, vol. 29, no. 7, pp. 1645-1660, 2013.

[2] L. Atzori, A. Iera, and G. Morabito, "The internet of things: A survey," Computer networks, vol. 54, no. 15, pp. 2787-2805, 2010.

[3] D. Cook and S. Das, Smart environments: Technology, protocols and applications. John Wiley \& Sons, 2004, vol. 43.

[4] G. C. Carter, "Coherence and time delay estimation," Proceedings of the IEEE, vol. 75, no. 2, pp. 236-255, 1987.

[5] W. Liu and S. Weiss, Wideband beamforming: concepts and techniques. John Wiley \& Sons, 2010, vol. 17.

[6] W. C. Chew, Waves and Fields in Inhomogeneous Media. New York: IEEE Press, 1995.

[7] G. Lerosey, J. De Rosny, A. Tourin, A. Derode, G. Montaldo, and M. Fink, "Time reversal of electromagnetic waves," Physical review letters, vol. 92, no. 19, p. 193904, 2004.

[8] T. Kaiser and F. Zheng, "Ultra wideband systems with MIMO," John Wiley and Sons, 2010.

[9] T. S. Rappaport et al., Wireless communications: principles and practice. Prentice Hall PTR New Jersey, 1996, vol. 2. 